\title{
The Importance of Factors Influencing the Brain Drain of IT Employee
}

\author{
Li Guo \\ Energy Economy and Management Research Center, \\ Xi'an University of Science and Technology, \\ Xi'an, 710054, China \\ E-mail: Christie_guo@163.com
}

\begin{abstract}
The paper builds a hierarchical model to analyze the factors influencing the brain drain of information technology (IT) employee based on the research result of domestic and foreign scholars. By using the questionnaire and analytic hierarchy process (AHP), the paper further investigates the importance of factors influencing the brain drain of IT employee. The results show that factor about company itself is the most important and the factor about employee is the least important in all factors. And factors such as industry environment, career development prospects, corporate culture, nature of work, total rewards are the most important factors influencing the brain drain of IT employee. The family status, age, educational background, seniority, lifestyle and working conditions are the least ones.
\end{abstract}

Keywords-Brain drain; AHP; Importance

\section{INTRODUCTION}

With the continuous development of science and technology in China and the introduction of foreign science and technology and capital, China gradually formed a situation of domestic competition internationalization, international competition localization. With a new round of the upsurge of the flow of talented people, many companies, especially many domestic IT enterprises are facing a serious problem: it is hard to retain talents. The loss of excellent talents has caused a crisis to some enterprises, and the crisis of talent has posed a major threat to the long-term development of these enterprises. How to prevent brain drain, has become a problem which many domestic IT enterprises management have to face and solve. According to analyze the importance of the reason of IT enterprise brain drain, this paper could find out the main factors influencing IT brain drain, and provide a reference for IT human resource management.

\section{LITERATURE REVIEW}

IT is the Information Technology in English, that is, the meaning of the information industry, mainly referring to communication industry, hardware and software industry, manufacturing industry and the relevant service industry [1]. The flow of the talents as a social phenomenon is objective to exist at all times and in all countries. American scholar Katz (Katz), in his theory about the life of organization, demonstrates the importance of the brain drain from the perspective of maintaining the vitality of the organization [2]. The outflow of talents refers to people specialized in certain

\author{
Chao-Lu Wu \\ Xi' an University of Science and Technology, \\ Xi'an, 710054, China \\ E-mail: 1037312087@qq.com
}

groups, organization and fields or other people with talents, leave their former attached or served target and transfer to other groups, organizations and fields. It usually called "Brain Drain" in the internationally [3]. The brain drain of the high-technology enterprise is the phenomenon that resulting in the talents needed in high-technology enterprises left the enterprise to find another job in some ways, because of various reasons. At the moment of current intense market competition, the brain drain is a terrible shock to the high-technology enterprise.

Wen-shuan Ling (2003) divided the factors influencing the brain drain from three levels which are macro, middle and micro [4]. The study of Mian Zhang(2003) pointed out that the traditional Chinese culture also affect the brain drain, which is the unique factor of domestic employee turnover [5], and it would have a lot guidance and reference meaning to the domestic scholar to do the further research in related field. Shu-jie Ma (2003) divided the factors of brain drain into three levels: social level, organizational level, individual level. [6] Chun-hui Lou (2004) believed that there are the factors affecting the brain drain mainly in the following areas: macro-environmental factors, and organizational and work-related factors, and personal factors [7]. Wen-hui Pang (2011) studied the elements influencing the brain drain mainly from the following three aspects: the external factor analysis, internal factors analysis and individual factors [8].

Although scholars have made a lot of analysis on the causes of brain drain, most researches are similar, whose contents mostly enumerate a variety of factors and the explanations of them, but the researches did not analyze the impact of these factors on the brain drain, so people cannot know which factor is the main cause of employee turnover, so, this paper uses the questionnaire survey and AHP (Analytic Hierarchy Process) method to analyze and investigate the importance of the reason of employee turnover.

\section{THE DESIGN OF THE RESEARCH}

Based on the literature review, according to Chinese scholars Cai-qin Li whose conclusions on the analysis of the reasons of the brain drain of IT employee[9], combining the research achievements of Yi-qun Fu, Shu-jie Ma, Ming Zeng and so on, hierarchical model of factors influencing the brain drain of IT employee is established, as shown in Figure 1. 


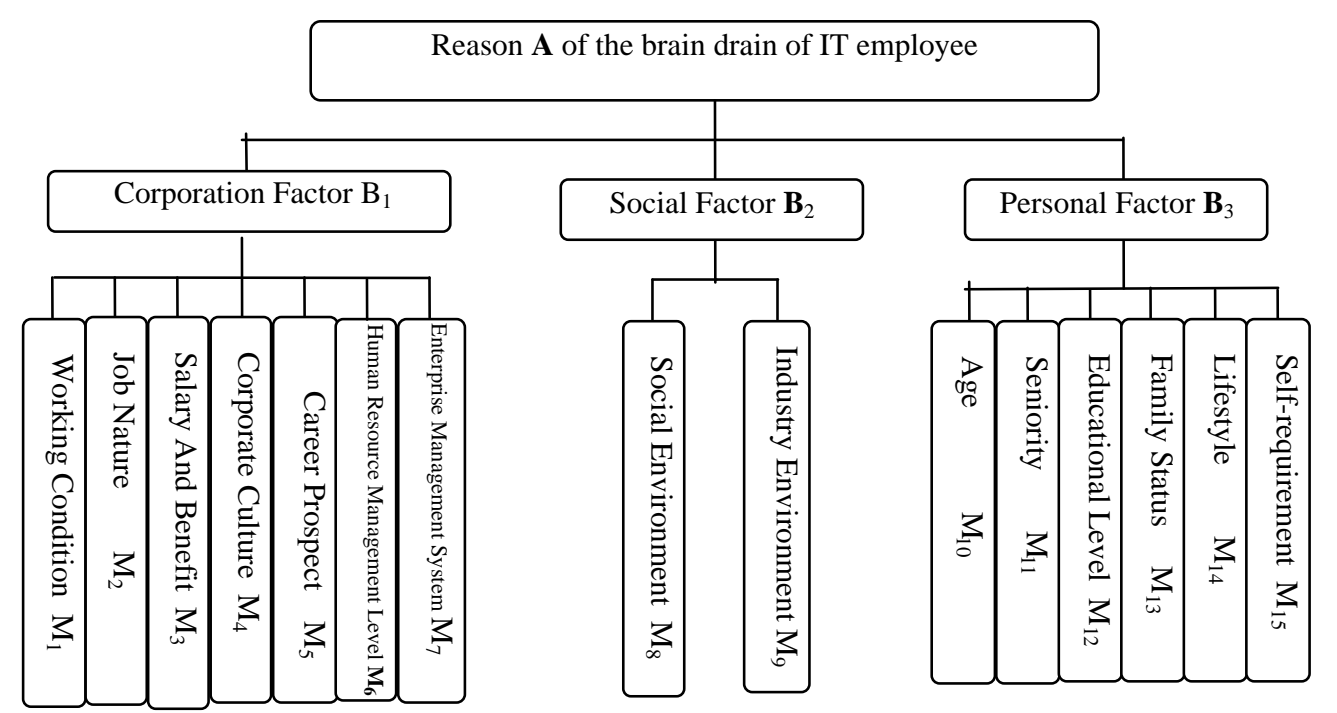

Figure.1. Hierarchical Model of Factors Influencing the Brain Drain of IT Employee

This research used questionnaire survey method, the purpose of the questionnaire survey is to study the factors influencing IT enterprise brain drain, judge the relative importance of each factor, and further explore the main reason for the influence of the loss of talents in IT enterprises by investigating IT staff of different enterprise through questionnaire surveys. This questionnaire is divided into three parts: the first part is the base information of the interviewees, which are sex, age and seniority; the second part is the definition or annotation of each influenced factor, in order to help the people who fill in the questionnaire understand the contents of each factor more comprehensive and deeper; and the third part is the analysis of the influenced factor. List the model of the two layers of factors separately, and then determine the relative importance between the relative factors one by one, through pair wise comparison on the relative factors.

Under the guidance of the principles of representativeness, measurability, objectivity, the data mostly was collected from 15 IT companies of Xi'an, whose annual sales are more than 100 million evenly, and their scale is pretty large. The target object of the survey is the IT enterprise employees at all levels. The process of data collection was completed under the support and cooperation of the human resources department of each company. Before the survey, the author and the relative person of human resources randomly selected investigated respondents together, in accordance with the standards, identified 160 staff as the subjects in 20 enterprises. Questionnaire survey used the form of on-site distribution and limited-time recycling, after filling in the questionnaire; the interviewee put the questionnaire into the envelope and delivered it to the author directly or returned to the author after each company's human resources department centrally collected. After repeated communication with the employees of the enterprise human resources department (which contains repeated collection), eventually 144 questionnaires were received, and the recovery was $90.00 \%$. Screened the recycled questionnaires again, those whose option could not meet the requirement were regarded as invalid questionnaires. There were 139 actual effective questionnaires, and the valid returned rate was $86.87 \%$. The following data analysis all use 139 as the actual sample size.

\section{DATA ANALYSIS AND RESULTS}

\section{A. Reliability and Validity Analysis}

Initially, analyze the reliability and validity of the data collected from the questionnaires. Cronbach alpha coefficient was used as the reliability test standard, after calculation, the Cronbach alpha coefficient was all above 0.78 , which showed that the questionnaire had good or very good internal consistency, and the reliability of the questionnaire could be accepted. Validity test used exploratory factor analysis, after calculation, the load of the element of the measured variables was all above 0.6, therefore, the construct validity of the questionnaire in this study could be accepted.

\section{B. The Importance of Factors Influencing the Brain Drain of IT Employee Based on AHP}

All the relevant data of all valid questionnaires were done statistics, and the method of weight average on the important degree score for each comparison program and the corresponding number of people was used to work out the corresponding numerical value in the judgment-matrix, and EXCEL was used to work out the final result, and finally the consistency test was carried out. The results are shown as following: (Table 1 to Table 5). 
TABLE I. CALCULATION AND THE CONSISTENCY TEST OF THE FIRST LAYER

\begin{tabular}{cccccccc}
\hline $\mathbf{A}$ & $\mathbf{B}_{\mathbf{1}}$ & $\mathbf{B}_{\mathbf{2}}$ & $\mathbf{B}_{\mathbf{3}}$ & $\mathbf{W}_{\mathbf{i}}$ & $\mathbf{W}_{\mathbf{i}}^{\mathbf{0}}$ & $\boldsymbol{\lambda}_{\mathrm{mi}}$ & Consistency Test \\
\hline $\mathbf{B}_{\mathbf{1}}$ & 1 & 3 & 7 & 2.006 & 0.669 & 3.014 & $\lambda_{\max }=3.007$ \\
$\mathbf{B}_{\mathbf{2}}$ & $1 / 3$ & 1 & 3 & 0.729 & 0.243 & 3.005 & $\mathrm{CI}=0.004 、 \mathrm{RI}=0.520$ \\
$\mathbf{B}_{\mathbf{3}}$ & $1 / 7$ & $1 / 3$ & 1 & 0.265 & 0.088 & 3.002 & $\mathrm{CR}=0.007<0.1$ \\
\hline
\end{tabular}

TABLE II. Calculation and the Consistency Test of B1

\begin{tabular}{cccccccccccc}
\hline $\mathbf{B}_{\mathbf{1}}$ & $\mathbf{M}_{\mathbf{1}}$ & $\mathbf{M}_{\mathbf{2}}$ & $\mathbf{M}_{\mathbf{3}}$ & $\mathbf{M}_{\mathbf{4}}$ & $\mathbf{M}_{\mathbf{5}}$ & $\mathbf{M}_{\mathbf{6}}$ & $\mathbf{M}_{\mathbf{7}}$ & $\mathbf{W}_{\mathbf{i}}$ & $\mathbf{W}_{\mathrm{i}}^{\mathbf{0}}$ & $\boldsymbol{\lambda}_{\mathrm{mi}}$ & Consistency Test \\
\hline $\mathbf{M}_{\mathbf{1}}$ & 1 & $1 / 5$ & $1 / 3$ & $1 / 7$ & $1 / 5$ & $1 / 5$ & $1 / 3$ & 0.226 & 0.032 & 7.210 & \\
$\mathbf{M}_{\mathbf{2}}$ & 5 & 1 & 3 & $1 / 3$ & $1 / 5$ & 5 & 7 & 1.380 & 0.197 & 8.449 & \\
$\mathbf{M}_{\mathbf{3}}$ & 3 & $1 / 3$ & 1 & $1 / 7$ & $1 / 3$ & 3 & 1 & 0.592 & 0.085 & 7.221 & $\lambda_{\max }=7.675$ \\
$\mathbf{M}_{\mathbf{4}}$ & 7 & 3 & 7 & 1 & 1 & 3 & 3 & 1.765 & 0.252 & 7.915 & $\mathrm{CI}=0.113$ \\
$\mathbf{M}_{5}$ & 5 & 5 & 3 & 1 & 1 & 5 & 5 & 1.977 & 0.282 & 8.138 & $\mathrm{CR}=0.083<0.1$ \\
$\mathbf{M}_{\mathbf{6}}$ & 5 & $1 / 5$ & $1 / 3$ & $1 / 3$ & $1 / 5$ & 1 & $1 / 3$ & 0.515 & 0.074 & 6.887 & \\
$\mathbf{M}_{7}$ & 3 & $1 / 7$ & 1 & $1 / 3$ & $1 / 5$ & 3 & 1 & 0.545 & 0.078 & 7.906 & \\
\hline
\end{tabular}

TABLE III. Table 3 Calculation and the Consistency Test of B2

\begin{tabular}{ccccccc}
\hline $\mathbf{A}$ & $\mathbf{M}_{\mathbf{8}}$ & $\mathbf{M}_{\mathbf{9}}$ & $\mathbf{W}_{\mathbf{i}}$ & $\mathbf{W}_{\mathbf{i}}^{\mathbf{0}}$ & $\boldsymbol{\lambda}_{\mathbf{m i}}$ & Consistency Test \\
\hline $\mathbf{M}_{\mathbf{8}}$ & 1 & $1 / 7$ & 0.375 & 0.125 & 2.000 & $\lambda_{\max }=2.000$ \\
$\mathbf{M}_{9}$ & 7 & 1 & 2.625 & 0.875 & 2.000 & $\begin{array}{c}\mathrm{CI}=0.000, \mathrm{RI}=0.000 \\
\mathrm{CR}=0.000<0.1\end{array}$ \\
\hline
\end{tabular}

TABLE IV. Calculation and the Consistency Test of B3

\begin{tabular}{ccccccccccc}
\hline $\mathbf{B}_{\mathbf{1}}$ & $\mathbf{M}_{\mathbf{1 0}}$ & $\mathbf{M}_{\mathbf{1 1}}$ & $\mathbf{M}_{\mathbf{1 2}}$ & $\mathbf{M}_{\mathbf{1 3}}$ & $\mathbf{M}_{\mathbf{1 4}}$ & $\mathbf{M}_{\mathbf{1 5}}$ & $\mathbf{W}_{\mathbf{i}}$ & $\mathbf{W}_{\mathbf{i}}^{\mathbf{0}}$ & $\boldsymbol{\lambda}_{\mathbf{m i}}$ & $\begin{array}{c}\text { Consistency } \\
\text { Test }\end{array}$ \\
\hline $\mathbf{M}_{\mathbf{1 0}}$ & 1 & $1 / 3$ & $1 / 3$ & 3 & $1 / 3$ & $1 / 3$ & 0.516 & 0.086 & 6.154 & \\
$\mathbf{M}_{\mathbf{1 1}}$ & 3 & 1 & 3 & 3 & 1 & $1 / 3$ & 1.154 & 0.192 & 6.662 & $\lambda \mathrm{max}=6.440$ \\
$\mathbf{M}_{\mathbf{1 2}}$ & 3 & $1 / 3$ & 1 & 3 & $1 / 3$ & $1 / 5$ & 0.675 & 0.112 & 6.278 & C.I. $=0.088$ \\
$\mathbf{M}_{\mathbf{1 3}}$ & $1 / 3$ & $1 / 3$ & $1 / 3$ & 1 & $1 / 3$ & $1 / 5$ & 0.311 & 0.052 & 6.252 & C.R. $=0.070<0.1$ \\
$\mathbf{M}_{\mathbf{1 4}}$ & 3 & 1 & 3 & 3 & 1 & 1 & 1.371 & 0.229 & 6.565 & \\
$\mathbf{M}_{\mathbf{1 5}}$ & 3 & 3 & 5 & 5 & 1 & 1 & 1.974 & 0.329 & 6.730 & \\
\hline
\end{tabular}

\section{Results}

As the tables shown above shows, seen from the influenced factors of B level, corporation factor is the first factor influencing the brain drain of IT employees; followed by social factor; and the Personal Factor is the third. Seen from the influenced factors of $\mathrm{M}$ level, the influence of Industry environment,personal career prospect,corporate culture and job nature is more significant, and the influence of age, seniority, family status and other factors is not significant to the brain drain of IT employee. 
TABLE V.

The Importance of Factors Influencing the Brain Drain of IT Employee

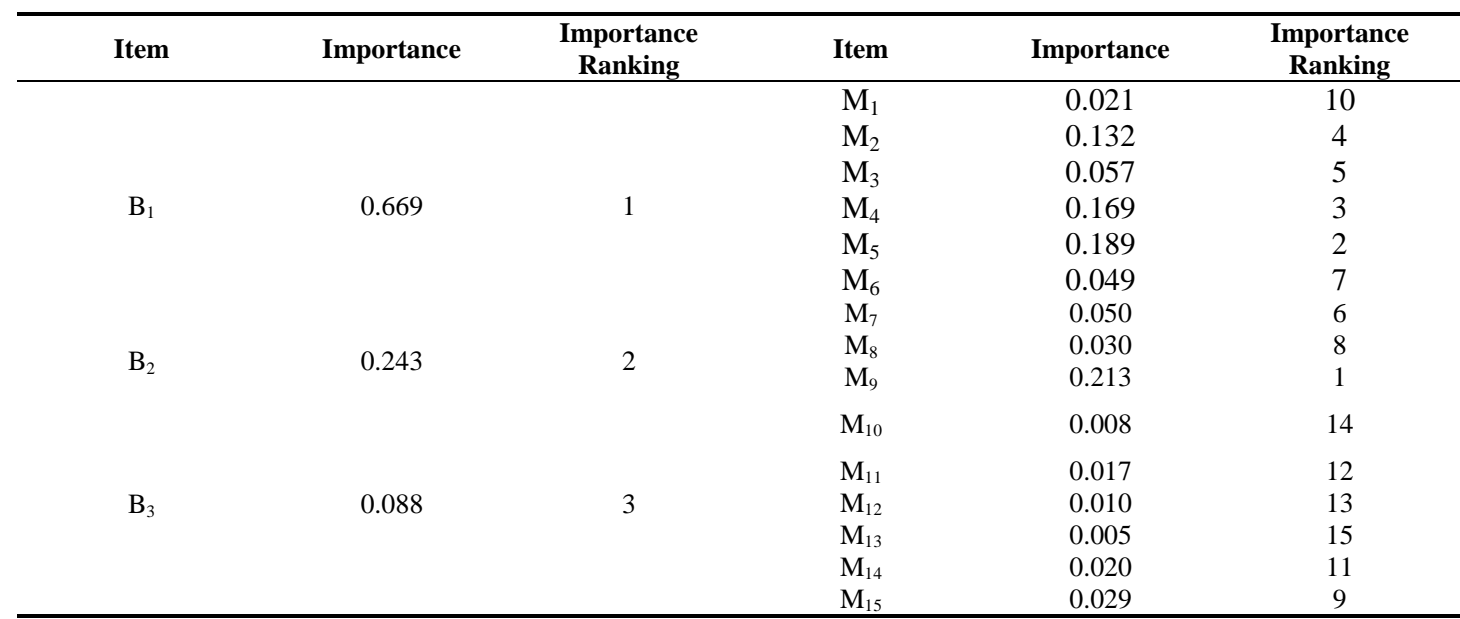

\section{CONCLUSIONS}

This study explores the influence of personal factor, cooperation factor and social factor for the brain drain of IT enterprise, and based on the method of analytic hierarchy process, analyzes specifically the degree of the influence of working condition, job nature, salary and benefit, corporate culture, career prospect, human resource management level, enterprise management system, social environment, industry environment, age, seniority, educational level, family status, lifestyle, self-requirement and other factors for the brain drain of IT enterprise. This research demonstrates: (1) Among cooperation factor, social factor and personal factor, the influence of corporation factor for brain drain of IT employee is the maximum, followed by social factor, and that of personal factor is the minimum; (2)The degree of the influence of industry environment, career prospect, corporate culture, job nature, salary and benefits for the brain drain of IT employee is heavy, however that of the influence of family status, age, educational level, seniority , lifestyle and working conditions and other factors is small.

Therefore, in order to reduce the loss of talents, initially, IT enterprises should start from their own, and pay attention to the construction of corporate culture. Corporate culture is the soul of an enterprise, creating the harmonious, equal and positive corporate culture from the physical layer, system layer and spiritual level can build a powerful spiritual pillar for the employees, enhance the cohesion and centripetal force of enterprises to make enterprises and employees form an effective community of life, and it could achieve that to develop the capability and potential of the employees continually, and to improve the passion of the hard-work of the employees and the purpose and effect of conviction.

Subsequently, help the employees to make their career planning. The enterprise according to the interest and specialty of the staff and the needs of the company can look for the best starting point between the employees and the idea and the goal of the enterprise to open several career ways for the staff, to excavate their potential fully, so that the enterprise really "make the best possible use of men and material". For those IT employees who have the desire of the management and the ability of talent, the enterprise can do a series of management knowledge training and practical practice, to help them step onto the manager's post. For those IT employees who want to improve their professional technology, the enterprise can establish the enterprise internal technology level system associated with the social unified technology level system, to broaden their personal development space.

Thirdly, perfect the performance management and salary management system. IT enterprises should establish a system of performance management system, adhere to the principle of openness, institutionalization and timely feedback, and administrate the ability and performance of the staff through open and fair scientific method to the characteristics of different positions. In a sense, salary not only represents money, but also is a reflection of the ability and quality of a person, and the self-worth of a person. Therefore, the enterprise should set up the salary system matched with the individual ability and the performance of the staff, because of the objective which enables private initiative. Moreover, IT companies could also according to their actual situation, adopt the buffet-style welfare plans, work out the workable combination of welfare plans to give a choice to the employees, which enables employees to actively participate in their determination of their own welfare form and content.

\section{ACKNOWLEDGEMENT}

This research was financially supported by the Project of Shaanxi Provincial Department of Education Research Funds (15JK1457), Soft Science Research Project of Shaanxi Province (2012KR2-04) and Philosophy and Social Sciences Prosperity Development Project of Xi'an University of Science and Technology (2014SX08).

\section{REFERENCES}

[1]. Deng Shou-peng, "Development trends of information technology and industry", communications market, vol.1, pp.30, 2003. 
[2]. Lin Lin. "The harm and the research of strategy caused by the loss of staff in small and medium enterprises", market modernization, vol.27, pp.131, 2015.

[3]. Niu Jing. "The status and the research of strategies on the recruitment outsourcing of technology talent in Beijing high and new technology enterprise", .human resources management, vol12, pp.230, 2015.

[4]. Ling Wen-shuan, Fu Yi-qun, Fang Li-luo, "The factors influencing the turnover intention of Employee", enterprise human resources management, vol8, pp.34, 2003.

[5]. Zhang Zhang, Zhang De, "The study of the factors influencing IT departing employee", China soft science, vol5, pp.71-80, 2003.
[6]. Ma Shu-jie, Chen Jing-qiu, Wang Lei, "Research on the reason of employee turnover". Development of China human resources, vol.9, 2003, pp.18-20, 2003.

[7]. Lou Chun-hui, "The factors of employee voluntary turnover and the analysis of its cost mode", commercial era, vol.21, pp.27, 2004.

[8]. Pang Wen-hui, "The analysis of the influencing factors of the knowledge-type employee turnover and the study of strategies", economic research guide, vol.2, pp.58-59, 2011.

[9]. Li Cai-qin, "Research on brain drain of Chinese IT industry. Dong Bei University of Finance and Economics", pp.18-20, 2007. 